\title{
Serological screening of coeliac disease: choosing the optimal procedure according to various prevalence values
}

\author{
G Corrao, G R Corazza, M L Andreani, P Torchio, R A Valentini, G Galatola \\ D Quaglino, G Gasbarrini, F di Orio
}

\section{Abstract}

The aim of this study was to select the best approach for screening coeliac disease patients among populations with different grades of disease prevalence. The diagnostic performance was assessed of class $A$ and $G$ antigliadin antibodies and class $A$ antiendomysium antibodies in 93 consecutive outpatients with suspected malabsorption, 44 of whom $(47 \%)$ had coeliac disease according to duodenal histological tests. Class G antigliadin antibodies provided the worst diagnostic values, whereas a high diagnostic validity was found for the other two tests. The positive predictive value corrected for the disease prevalence expected in coeliac disease relatives (5\%) and the general population $(0 \cdot 2 \%)$ fell to $30 \%$ and $<2 \%$ respectively for class $A$ antigliadin antibodies, whereas it remained $100 \%$ for antiendomysium antibodies in both situations, providing an optimal value for their use as a screening test and as a valid alternative to duodenal biopsy when this is not feasible. The high cost of antiendomysium antibodies and the invasive nature of duodenal biopsy prevent them being used widely as screening procedures. A cost effective two step approach was simulated measuring class $A$ antigliadin antibodies in all subjects of the target population (first step), and performing a confirmation test (antiendomysium antibodies or duodenal biopsy) only in subjects positive for antigliadin antibodies. The results show that such a procedure should be recommended only for subjects with an expected low disease prevalence - that is, $5 \%$ for coeliac disease relatives and $0.2 \%$ for the general population - as the positive predictive value was always $100 \%$ with an acceptable false negative rate $(6 \%$ and $11 \%$ respectively), irrespective of which of the two confirmation tests was used. This approach avoids the use of the confirmation test in $63 \%$ and $89 \%$ of subjects respectively for the two levels of prevalence, resulting in a considerable reduction of the cost. Patients seen for suspected malabsorption with an expected high prevalence of coeliac disease should not have such a serological screening procedure. In conclusion, antigliadin antibodies are useful to screen for asymptomatic coeliac disease in non-hospital communities if antiendomysium anti- bodies are used as a confirmation test: the latter is a reasonable valid alternative to duodenal biopsy.

(Gut 1994; 35: 771-775)

Coeliac disease is a condition characterised by villous atrophy of the small intestine induced in predisposed subjects by the ingestion of gluten containing gliadins and prolamins. Its prevalence in European countries based on symptomatic cases, has been calculated to be between $1: 300$ and $1: 1000$ subjects. ${ }^{12}$ The classic clinical presentation, however, with weight loss and diarrhoea ${ }^{34}$ is now not often encountered, thus the diagnosis of coeliac disease is often missed, ${ }^{5}$ and its actual prevalence is probably higher.

The definitive diagnosis of coeliac disease is based on finding specific histological hallmarks on jejunal or duodenal biopsy specimens. ${ }^{6}$ Serum antigliadin antibodies (AGA) and antiendomysium antibodies (EMA) have been found to provide specific and sensitive tests for identifying patients with coeliac disease. ${ }^{78}$ The diagnostic performance, however, of such tests has not yet been clarified because previous studies aimed only at defining their best cut off value. This fact is a limit to their use and to the interpretation of their results. ${ }^{9}$

Furthermore, the actual predictivity of a test may vary according to the prevalence of the disease that the test should diagnose. We have reasoned that, on the one hand, the high predictive values reported for AGA in diagnosing coeliac disease may fall when these tests are implemented as a screening procedure in subjects with low prevalence of coeliac disease, making them unsuitable for this purpose. On the other hand, the high cost of EMA is itself a limiting factor for its wide use in screening programmes.

Thus, our aims were: (1) to estimate the diagnostic performance of AGA and EMA for diagnosing coeliac disease; (2) to estimate the diagnostic performance of various combinations of tests, to find out if we could identify an optimal two step screening procedure for coeliac disease in subjects with progressively decreasing values of disease prevalence. The procedure used the cheaper test with high sensitivity as a first step and the most expensive but highly predictive test as the confirmation test in patients selected as positive by the first step test. For this purpose we used the receiver operating characteristic analysis, which evaluates the performance of a diagnostic or screening test, and calculates cut off 
values taking into account both the prevalence of the disease in various target populations and the comparative weight of misclassification error. ${ }^{10}$

\section{Suijjects and methods}

\section{PATIENTS}

During the period September 1990 to December 1991 we studied 93 consecutive patients (24 males, 69 females; mean age 39 years, range 14-71) referred to the outpatient clinic of the I Department of Medical Pathology at the University of Bologna, Italy for clinically suspected coeliac disease. Coeliac disease was diagnosed in 44 patients according to the classic duodenal histological findings. After extensive diagnostic examination that included duodenal biopsy in all patients, the remaining final diagnoses were: Crohn's disease $(n=7)$, ulcerative colitis $(n=5)$, small intestine bacterial overgrowth $(n=3)$, giardiasis $(n=3)$, Whipple's disease $(n=2)$, small intestine lymphoma $(n=1)$, gastric lymphoma $(n=1)$, and irritable bowel syndrome $(n=27)$.

\section{SEROLOGICAL ANALYSIS}

A serum sample was collected from all patients at the time of their first visit to the clinic and then stored at $-20^{\circ} \mathrm{C}$. AGA and EMA were measured in the serum using the same methods throughout the study, by a single pathologist who did not know the diagnoses. Class $A$ and $G$ AGA (IgA-AGA, IgG-AGA) were measured using a previously described microenzyme linked immunosorbent assay (ELISA). ${ }^{7}$ Briefly, $200 \mu \mathrm{l}$ serum diluted samples were added to microtitre plates (Dynatech, Billinghurst, UK) precoated with commercial crude gliadin suspension incubated at $20^{\circ} \mathrm{C}$ for three hours. After washing, alkaline phosphatase conjugated antihuman IgA and IgG were added and the plates incubated again at room temperature for three hours, then washed. The reaction was detected by reading the absorbance at $405 \mathrm{~nm}$ after addition of a substrate solution of $p$-nitrophenyl-phosphate in diethanolamine magnesium chloride buffer.

IgA-EMA were detected using an indirect immunofluorescence technique ${ }^{11}$ on commercial sections of monkey oesophagus (Biosystems, Milan, Italy). IgA specific for the endomysial lining of myofibrils were identified by their reticulin like staining of smooth muscle bundles. Serum samples containing antibodies at any titre were considered positive.

PREDICTIVE ABILITY OF IgA-AGA AND IgG-AGA The receiver operating characteristic analysis was used to compare the predictive ability of IgA-AGA and IgG-AGA. This analysis summarises the possible sets of $2 \times 2$ classification tables that result when the cut off point is varied from the smallest to largest possible figure. ${ }^{12}$ The model plots sensitivity (the proportion of coeliac disease patients attributed by the test to the coeliac group) $v$ 1-specificity (the proportion of control patients attributed by the test to the coeliac group) for each possible value of the test. The receiver operating characteristic curve shows the discriminative ability of the screening test. The curve for a perfect test is as far upward and to the left as possible; a diagonal line is indicative of a non-informative test. The area under the receiver operating characteristic curve is considered the best index for detection. ${ }^{13} 14$ The area under curve represents the probability that the test correctly classifies two randomly paired normal and coeliac disease subjects. ${ }^{15} 16$ Several methods have been proposed for estimating area under curve, according to the underlying distributions. ${ }^{1517}$ In this study, we used a method based on the maximum likelihood estimate, ${ }^{15}$ developed as a statistical program (LABROC-1 and CLABROC) by Metz et al. ${ }^{18}$ Results were subsequently confirmed on a subset of the analysis using the non-parametric approach described by Delong et al. ${ }^{19}$

The receiver operating characteristic analysis was also carried out to find out if using the two tests simultaneously results in a greater discriminant ability than using either test alone. For this purpose, we obtained a discriminant function using a linear discriminant analysis model, where the dependent variable was the diagnostic category (presence or absence of coeliac disease) and the independent variables were serum IgA-AGA and IgGAGA. The receiver operating characteristic curve was obtained from the a posteriori probability of the discriminant function, and area under curve was calculated as described above.

\section{CHOICE OF THE SCREENING PROCEDURE}

The performance of a screening test should be assessed in empirical conditions, where half of the sample carries the disease and the other half does not. For any cut off point of the test, the predictive ability is affected by the prevalence of the disease in the target population, thus, whenever the test is applied to a community sample, an adjustment for the prevalence should be considered. ${ }^{10}$ Furthermore, utilising a single test for screening purposes often results in a poor test performance. For this reason, in our study we considered the use of a first step screening test applied to the whole sample, and a confirmation second step test applied to subjects positive to the first step test. Because detection of AGA is less costly and easier to perform than detection of EMA, we used the first as the first step test and the second as the confirmation test. We simulated the impact of this procedure in various conditions, by looking at: (a) the first step test (estimates of receiver operating characteristic validity at different AGA cut off points were considered); (b) the conformation test (biopsy $v$ IgA-EMA); (c) various target populations in which the prevalence of coeliac disease would vary.

The predictivity of such a procedure has 
TABLE I Distribution of coeliac and control patients according to various classes of $\operatorname{Ig} A-A G A$ and $\operatorname{Ig} G-A G A$ values

\begin{tabular}{|c|c|c|c|c|}
\hline \multirow[b]{2}{*}{$\begin{array}{l}\text { ELISA } \\
\text { units }\end{array}$} & \multicolumn{2}{|l|}{$\operatorname{Ig} A-A G A$} & \multicolumn{2}{|l|}{$\operatorname{Ig} G-A G A$} \\
\hline & $\begin{array}{l}\text { Coeliac } \\
\text { patients } \\
\text { no (\%) }\end{array}$ & $\begin{array}{l}\text { Control } \\
\text { patients } \\
\text { no }(\%)\end{array}$ & $\begin{array}{l}\text { Coeliac } \\
\text { patients } \\
\text { no }(\%)\end{array}$ & $\begin{array}{l}\text { Control } \\
\text { patients } \\
\text { no (\%) }\end{array}$ \\
\hline $0 \cdot 0-0 \cdot 2$ & $4(9 \cdot 1)$ & $41(83 \cdot 7)$ & $11(25 \cdot 0)$ & $42(85 \cdot 7)$ \\
\hline $0 \cdot 2-0 \cdot 4$ & $2(4 \cdot 5)$ & $8(16 \cdot 3)$ & $3(6 \cdot 8)$ & $1(2 \cdot 0)$ \\
\hline $0.4-0.6$ & $1(2 \cdot 3)$ & $0(-)$ & $7(15.9)$ & $1(2 \cdot 0)$ \\
\hline $0 \cdot 6-0.8$ & $2(4 \cdot 5)$ & $0(-)$ & $2(4 \cdot 5)$ & $2(4 \cdot 1)$ \\
\hline $0 \cdot 8-1 \cdot 0$ & $4(9 \cdot 1)$ & $0(-)$ & $2(4 \cdot 5)$ & $1(2 \cdot 0)$ \\
\hline $1 \cdot 0-2 \cdot 0$ & $12(27 \cdot 3)$ & $0(-)$ & $13(29 \cdot 5)$ & $1(2 \cdot 0)$ \\
\hline $2 \cdot 0-3 \cdot 0$ & $4(9 \cdot 1)$ & $0(-)$ & $1(2 \cdot 3)$ & $1(2 \cdot 0)$ \\
\hline $3 \cdot 0-4 \cdot 0$ & $5(11 \cdot 4)$ & $0(-)$ & $1(2 \cdot 3)$ & $0(-)$ \\
\hline$\geqslant 4 \cdot 0$ & $10(22 \cdot 7)$ & $0(-)$ & $4(9 \cdot 1)$ & $0(-)$ \\
\hline Total & $44(100 \cdot 0)$ & $49(100 \cdot 0)$ & $44(100 \cdot 0)$ & $49(100 \cdot 0)$ \\
\hline
\end{tabular}

TABLE II Sensitivity and specificity of $\operatorname{Ig} A-A G A$ for various cut off points estimated by the fitted receiver operating characteristic curves

\begin{tabular}{lll}
\hline Cut off (ELISA units) & Specificity & Sensitivity \\
\hline 0.08 & 0.66 & 0.96 \\
0.09 & 0.75 & 0.95 \\
$0 \cdot 10$ & 0.83 & 0.93 \\
$0 \cdot 12$ & 0.89 & 0.91 \\
0.17 & 0.96 & 0.88 \\
0.39 & 0.99 & 0.79 \\
0.52 & 1.00 & 0.70 \\
\hline
\end{tabular}

been estimated under the assumption that the validity of serological tests for coeliac disease is independent of the presence or absence of symptoms.

\section{Results}

IgA-AGA AND IgG-AGA

Table 1 shows the distribution of coeliac disease and control patients according to the results of IgA-AGA and IgG-AGA. All 49 control patients and six coeliac disease patients $(13.6 \%)$ had IgA-AGA below 0.4 ELISA units. Forty three control patients $(87 \cdot 8 \%)$ and 14 coeliac disease patients $(31 \cdot 8 \%)$ had IgGAGA values below this threshold.

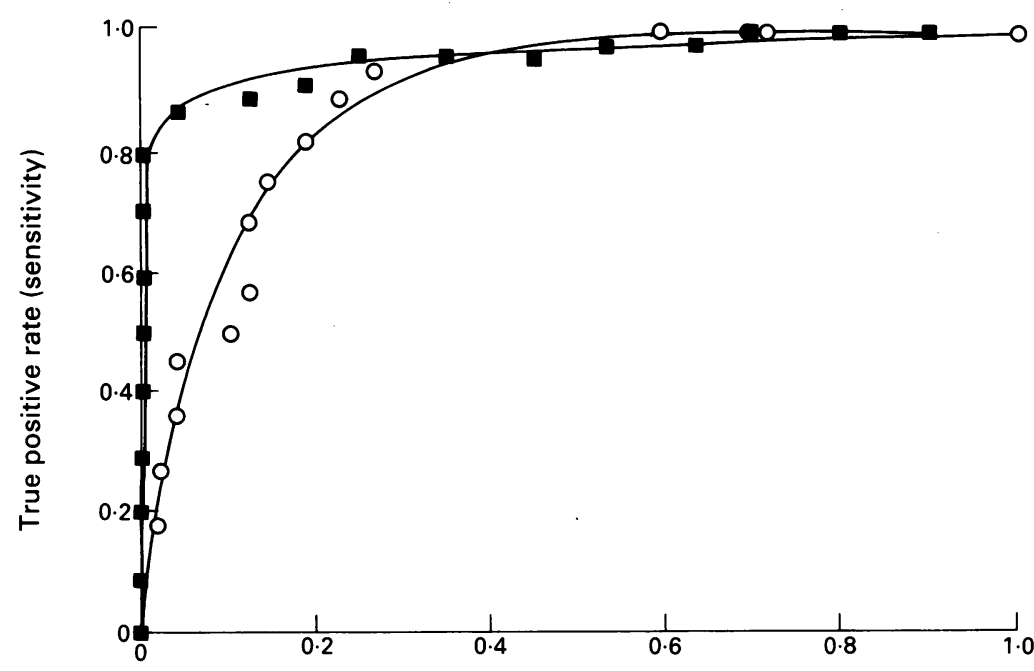

False positive rate (1-specificity)

Empirical points and fitted curves showing receiver operating characteristic curves for IgA-AGA (closed squares) and IgG- $A G A$ (open circles). The curve relative to Ig $A-A G A$ $\operatorname{Ig} A-A G A$ (closed squares) and IgG- $A G A$ (open circles). The curve relative to IgA- $A G A$
is shifted leftward, showing improved sensitivity and a reduced false positive rate of the test in the discrimination between coeliac disease and control patients.
TABLE III Positive and negative predictive values of IgA$A G A$ according to various cut off points and various prevalence values of coeliac disease

\begin{tabular}{lllll}
\hline \multirow{2}{*}{$\begin{array}{l}\text { Cut off } \\
\text { (ELISA units) }\end{array}$} & Predictivity & $47 \%$ & $5 \%$ & $0.2 \%$ \\
\cline { 3 - 5 } & PPV & 0.71 & 0.13 & 0.0056 \\
0.08 & NPV & 0.95 & 1.00 & 0.9998 \\
& PPV & 0.83 & 0.22 & 0.0108 \\
& NPV & 0.93 & 1.00 & 0.9998 \\
0.12 & PPV & 0.88 & 0.30 & 0.0163 \\
& NPV & 0.91 & 0.99 & 1.0000 \\
\hline
\end{tabular}

PPV = positive predictive value NPV $=$ negative predictive value.

TABLE IV Distribution of IgA-EMA titres in coeliac and control patients

\begin{tabular}{lcl}
\hline $\begin{array}{l}\text { Antibodies } \\
\text { titre }\end{array}$ & $\begin{array}{l}\text { Coeliac patients } \\
(n=44)\end{array}$ & $\begin{array}{l}\text { Control patients } \\
(n=49)\end{array}$ \\
\hline Negative $(1: 1)$ & 1 & 49 \\
Positive $(1: 5)$ & 5 & 0 \\
Positive $(1: 50)$ & 2 & 0 \\
Positive $(1: 100)$ & 1 & 0 \\
Positive (1:200) & 5 & 0 \\
Positive (1:500) & 30 & 0 \\
\hline
\end{tabular}

The Figure shows the receiver operating characteristic curves for IgA-AGA and IgGAGA. The best predictive ability was found for IgA-AGA. The area under curve values (SE) were $0.9599(0.0214)$ for IgA-AGA and $0.8933(0.0327)$ for IgG-AGA, the two values being significantly different $(z=3 \cdot 18$; two tailed $p$ value $=0.0015)$. The area under curve obtained by the a posteriori probability according to the linear discriminant analysis model was $0.9611(0.0297)$, and it did not differ from the area under curve of $\operatorname{IgA}-\mathrm{AGA}(\mathrm{z}=0 \cdot 18$; two tailed $p$ value $=0.8572$ ).

Table II shows that, for IgA-AGA, sensitivity decreases and specificity increases at increased cut off values.

Table III shows the positive and negative predictive values of IgA-AGA calculated for three cut off points, simulating three different prevalence values of coeliac disease. The positive predictive value increases at increased cut off points and at increased prevalence values. The best cut off point (positive predictive value $=88 \%$ ) was $0 \cdot 12$ ELISA units in the setting of a $47 \%$ prevalence of coeliac disease; poorer positive predictive values were seen for lower prevalence values. The negative predictive value was high throughout the simulation, the worse figure $(91 \%)$ being seen at the 0.12 ELISA units cut off point in the case of the highest prevalence of coeliac disease.

EMA-IgA

Table IV shows the distribution of control and coeliac disease patients according to the IgA-EMA titres. In all 49 controls and one coeliac disease patient no IgA-EMA were detectable in the serum. The serum samples in which IgA-EMA were detectable were considered as positive, thus sensitivity was $97 \cdot 7 \%$ and specificity $100 \%$. At increased IgA-EMA dilutions, sensitivity decreased but specificity remained $100 \%$. 
TABLE $\mathrm{V}$ Sensitivity of the two step screening procedure according to various IgA-AGA cut off points and to the confirmation test used

\begin{tabular}{lll}
\hline $\begin{array}{l}\text { Cut off (ELISA units) } \\
\text { for first step test } \\
\text { (IgA-AGA) }\end{array}$ & $\begin{array}{l}\text { Confirmation } \\
\text { test }\end{array}$ & Sensitivity \\
\hline 0.08 & Biopsy & 0.96 \\
& IgA-EMA & 0.94 \\
0.10 & Biopsy & 0.93 \\
0.12 & IgA-EMA & 0.91 \\
& Biopsy & 0.91 \\
& IgA-EMA & 0.89 \\
\hline
\end{tabular}

TWO STEP PROCEDURE (IgA-AGA + IgA-EMA OR BIOPSY)

Both the specificity and positive predictive value of the two step procedure remained $100 \%$, because no false positive results resulted from duodenal biopsy (gold standard test) or IgA-EMA. Table V shows that sensitivity was greater when biopsy was used as the confirmation test, and when lower IgA-AGA cut off points were chosen.

Table VI shows high negative predictive value throughout the simulation, particularly in the case of a low prevalence of coeliac disease. The worse negative predictive value $(92 \%)$ was seen when IgA-EMA were assessed in subjects with higher than $0 \cdot 12$ ELISA units IgA-AGA, in the case of $47 \%$ prevalence of coeliac disease. The proportion of subjects positive for the first step test, in whom the second step confirmation test was carried out, was higher at increased values of disease prevalence and at decreased IgA-AGA cut off points.

\section{Discussion}

Early withdrawal from gluten intake in patients with coeliac disease prevents the complications resulting from long standing disease and reduces the incidence of gastrointestinal malignancies, which occur with higher incidence than expected in coeliac disease patients. ${ }^{20} 21$ The implementation of screening programmes to detect asymptomatic coeliac disease patients is, however, hampered by the fact that duodenal biopsy, which is the gold standard diagnostic procedure, is comparatively invasive and time consuming and cannot be used for some purposes, particularly in children.

The availability of improved techniques for measuring AGA in serum as markers for coeliac disease has provided the feasibility of screening programmes on wide series of high risk subjects ${ }^{22-27}$ and in the general

TABLE VI Negative predictive value and frequency of use of the comfirmation test in two step screening procedure according to various IgA-AGA cut off points and to the confirmation test used, in three different prevalence values of coeliac disease $(C D)$

\begin{tabular}{|c|c|c|c|c|c|c|c|}
\hline \multirow{3}{*}{$\begin{array}{l}\text { Cut off } \\
\text { (ELISA units) } \\
\text { for first step test } \\
(I g A-A G A)\end{array}$} & \multirow{3}{*}{$\begin{array}{l}\text { Confirmation } \\
\text { test }\end{array}$} & \multicolumn{6}{|c|}{ Expected prevalence of $C D$} \\
\hline & & \multicolumn{2}{|l|}{$47 \%$} & \multicolumn{2}{|l|}{$5 \%$} & \multicolumn{2}{|l|}{$0 \cdot 2 \%$} \\
\hline & & $N P V$ & $F R E Q$ & $N P V$ & $F R E Q$ & $N P V$ & $F R E Q$ \\
\hline 0.08 & $\begin{array}{l}\text { Biopsy } \\
\text { EMA }\end{array}$ & $\begin{array}{l}0.97 \\
0.94\end{array}$ & $0 \cdot 70$ & $\begin{array}{l}1.00 \\
1.00\end{array}$ & $0 \cdot 37$ & $\begin{array}{l}1 \cdot 00 \\
1.00\end{array}$ & 0.34 \\
\hline $0 \cdot 10$ & $\begin{array}{l}\text { Biopsy } \\
\text { EMA }\end{array}$ & $\begin{array}{l}0.95 \\
0.93\end{array}$ & 0.52 & $\begin{array}{l}1.00 \\
1.00\end{array}$ & $0 \cdot 21$ & $\begin{array}{l}1.00 \\
1.00\end{array}$ & $0 \cdot 17$ \\
\hline $0 \cdot 12$ & $\begin{array}{l}\text { Biopsy } \\
\text { EMA }\end{array}$ & $\begin{array}{l}0.93 \\
0.92\end{array}$ & 0.48 & $\begin{array}{l}1.00 \\
0.99\end{array}$ & $0 \cdot 15$ & $\begin{array}{l}1.00 \\
1.00\end{array}$ & $0 \cdot 11$ \\
\hline
\end{tabular}

$\mathrm{NPV}=$ negative predictive value; $\mathrm{FREQ}=$ use of the confirmation test. population. 2829 Different techniques have, however, been used in such studies and the lack of standardisation of the test and insufficient knowledge on its diagnostic ability, generates difficulty in choosing the optimal method and interpreting its result.

We have confirmed that IgA-AGA have a better discriminant ability than IgG-AGA, whatever cut off point is considered. ${ }^{30}$ The sequential use of both tests did not increase the discriminant ability of the single use of IgAAGA. For this reason, only IgA-AGA has been considered in the following analysis. Despite the fact that IgA-AGA were $91 \%$ sensitive and $89 \%$ specific, their positive predictive value corrected for a $0 \cdot 2 \%$ prevalence of coeliac disease was below $2 \%$, showing that this test is not suitable for screening coeliac disease in the general population.

The detection of serum EMA provides an alternative possibility for a sensitive and specific serological test for the diagnosis of coeliac disease. ${ }^{31}$ Our results confirm published works, showing that EMA provide $98 \%$ sensitivity and $100 \%$ specificity. ${ }^{8} 1131$ Furthermore, by contrast with our results on IgAAGA, the positive predictive value of EMA corrected for a $0.2 \%$ prevalence of coeliac disease remained $100 \%$. This represents the optimal prerequisite for a screening procedure to be used in the general population. Thus, EMA may become a valid non-invasive alternative to duodenal biopsy, particularly in children and where endoscopic facilities are not available. The high cost of this test, however, discourages its implementation on a wide scale.

Thus, to try and limit the use of EMA to a smaller selected population, we considered a two step procedure, using the cheaper and easier test (IgA-AGA) as a first step, and carrying out the most expensive but highly predictive confirmation test (EMA or biopsy) only in IgA-AGA positive subjects, simulating also three different conditions of coeliac disease prevalence.

When we considered a $47 \%$ prevalence, which was characteristic of subjects in our gastroenterology outpatient clinic, the positive predictive value according to an IgA-AGA cut off point of 0.08 ELISA units was $71 \%$. Because a duodenal biopsy is justified in all positive patients, screening with IgA-AGA would result in a $29 \%$ rate of negative biopsies, entailing an overall 3\% false negative rate and avoiding a negative biopsy in $29 \%$ of the cases. By increasing the IgA-AGA cut off point to $0 \cdot 12$ ELISA units, a greater proportion of biopsies will be avoided (52\%), but the diagnosis would be missed in $7 \%$ of the cases. Thus, considering the risk of missing the diagnosis of coeliac disease, we do not recommend such a procedure in a gastroenterology clinic, but suggest that a duodenal biopsy is carried out as the first and definitive test in all patients referred for suspected coeliac disease.

We then simulated the screening of asymptomatic high risk subjects, in whom coeliac disease prevalence should be between 2 and $8 \%{ }^{26} 27$ Considering an intermediate 5\% 
prevalence of coeliac disease, IgA-AGA at a 0.08 ELISA units cut off point resulted in $13 \%$ positive predictive value, and $100 \%$ negative predictive value. This setting cannot justify a duodenal biopsy in asymptomatic IgA-AGA positive subjects especially when it is expected that there is low compliance to the test by such subjects. The detection of EMA as a second step confirmation test in IgA-AGA positive subjects would be the optimal procedure, causing a negligible reduction of the overall sensitivity (from 96 to $94 \%$ ), but a considerable increase in specificity (from 66 to $100 \%$ ), entailing a $6 \%$ false negative rate and avoiding the EMA test in $63 \%$ of the cases.

When we considered a $0 \cdot 2 \%$ coeliac disease prevalence, simulating the expected prevalence in the general population, ${ }^{2}$ IgA-AGA at a 0.08 ELISA units cut off point resulted in a positive predictive value below $1 \%$. In IgAAGA positive subjects, duodenal biopsy is clearly not feasible, whereas detection of EMA is again the procedure of choice. If we used a higher cut off value for IgA-AGA (0.12 ELISA units), the detection of EMA resulted in $100 \%$ positive predictive value and a near $100 \%$ negative predictive value, entailing an $11 \%$ false negative rate, and avoiding EMA in $89 \%$ of the cases. This approach had the advantage of saving $87 \%$ of the cost by comparison with the use of EMA as a single step screening test.

The estimated diagnostic values of the two step procedure are affected by the design of our study, which used a sample of coeliac disease patients and a group of control patients with other gastrointestinal diseases. Because antigliadin antibodies can be found in patients with inflammatory bowel disease, ${ }^{32}$ we can hypothesise that the actual specificity of $\operatorname{IgA}$ AGA would have been higher than we estimated, had we considered asymptomatic patients or subjects from the general population. Thus, both the overall false negative rate and the frequency of use of costly confirmation tests, such as EMA, should be actually lower than we estimated in running a screening programme based on the two step procedure that we have examined.

In conclusion, we have shown that the best diagnostic approach to the diagnosis of coeliac disease using serological screening tests changes according to the target population. In a gastroenterology clinic we suggest a single step procedure (duodenal biopsy) in patients with clinically suspected coeliac disease. In screening asymptomatic high risk relatives or subjects in the general population we suggest the use of IgA-AGA followed by the detection of EMA only in IgA-AGA positive subjects. This approach represents a compromise between the poor predictivity of AGA, the high cost of EMA, and the invasive nature of duodenal biopsy.

1 Mylotte M, Egan-Mitchell B, McCarthy CF, McNicholl B. Incidence of coeliac disease in the West of Ireland. $B M$ 1973; 1: 703-5.

2 Logan RFA, Rifkind EA, Busuttil A, Gilmour HM, Ferguson A. Prevalence and 'incidence' of coeliac disease in Edinburgh and the Lothian region of Scotland. Gastroenterology 1986; 90: 334-42.
3 Logan RFA, Tucker G, Rifkind EA, Heading RC, Ferguson A. Changes in clinical features of coeliac disease in adults in Edinburgh and the Lothians, 1960-1979. BMF 1983; 286: $95-7$.

4 Corazza GR, Frisoni M, Treggiari EA, Valentini RA, Filipponi C, Volta U, et al. Subclinical celiac sprue. Increasing occurrence and clues to its diagnosis. $\mathcal{f}$ Clin Gastroenterol 1993; 16: 16-21.

5 Swinson CM, Levy AJ. Is coeliac disease underdiagnosed? BMF 1980; 281: 1258-60.

6 Rubin CE, Brandborg LL, Phelps PC, Taylor HC Jr. Studies of coeliac disease. I. The apparent identical and specific nature of the duodenal and proximal jejunal lesion in coeliac disease and idiopathic sprue. Gastroenterology 1960; 38: 28-34.

7 Volta U, Lenzi M, Lazzari R, Cassani F, Collina A, Bianchi FB, et al. Antibodies to gliadin detected by immunofluorescence and a micro-ELISA method: markers of active childhood disease. Gut 1985; 26: 667-71.

8 Ferreira M, Lloyd Davies S, Butler M, Scott D, Clark M, Kumar P. Endomysial antibody: is it the best screening Kumar P. Endomysial antibody: is it the best

9 Lorner A, Lebenthal E. The controversy of the use of anti-gluten antibody (AGA) as a diagnostic tool in celiac disease. f Pediatr Gastroenterol Nutr 1991; 12: 407-9.

10 Ritchie K, Fuhrev R. A comparative study of the performance of screening tests for senile dementia using receiver operating characteristics analysis. $\mathcal{F}$ Clin Epidemiol 1992; 45: $627-37$.

11 Chorzelski TP, Beutner EH, Suley J, Tchorzewska H, Jablonska S, Kumar V, et al. IgA antiendomysium antibody. A new immunological marker of dermatitis herpetiformis and coeliac disease. Br $\mathcal{f}$ Dermatol 1984; 111: 395-402.

12 Swets JA, Pickett RM, Whitehead SF. Assessment of diagnostic technologies. Science 1989; 205: 753-9.

13 Swets JA. Form of empirical ROC in discrimination and diagnosis: implications for theory and measurement of performance. Psychol Bull 1986; 99: 181-98.

14 Hanley JA. Receiver operating characteristics (ROC) metodology: the state of the art. Crit Rev Diagn Imaging 1989; 29: 307-34.

15 Dorfmann DD, Alf E. Maximum likelihood estimation of parameters of signal detection theory and determination of confidence intervals: rating method data. 7 Math Psychol 1969; 6: 487-96.

16 Hanley JA, MacNeil BJ. The meaning and use of the area under a receiver operating characteristics (ROC) curve. under a receiver operating
Radiology 1982; 143: 29-36.

17 Hanley JA, MacNeil BJ. A method of comparing the areas under receiver operating characteristics curves derived from the same cases. Radiology 1983; 148: 839-43.

18 Metz CE, Wang PL, Kranman HB. A new approach for testing the significance of differences between ROC curves measured from correlated data. In: Deconik, F, ed. Information processing in medical imaging: proceedings of the 8th conference. Amsterdam: Martinus Nijhof, 1984: 432-55.

19 Delong ER, Delong DM. Clarke-Pearson DL. Comparing the areas under two or more correlated receiver operating characteristics curves: a non parametric approach. characteristics curves: a non

20 Logan RT, Rifkind EA, Turner ID, Ferguson A. Mortality in celiac disease. Gastroenterology 1989; 97: 265-71.

21 Holmes JKT, Prior P, Lane MR, Pope D, Allan RN Malignancy in coeliac disease-effect of a gluten free diet. Gut 1989; 30: 333-7.

22 Cacciari E, Salardi S, Volta U, Biasco G, Lazzari R, Corazza GR, et al. Can antigliadin antibody test detect symptomless coeliac disease in children with short stature? Lancet 1985. i: 1469-71.

23 Savilhati E, Simell O, Koskimio S, Rilva A, Akerblom HK. Celiac disease in insulin-dependent diabetes mellitus. f Pediatr 1986; 108: 690-3.

24 Fornasieri A, Sinico RA, Maldifassi P, Bernasconi P, Vegni M, D'Amico G. IgA-antigliadin antibodies in IgA mesangial nephropathy (Berger's disease). BMF 1987; 295: 78-80.

25 O'Farrelly C, Melcher D, Price R, Sherwood R, Marten D, McDougall $\mathrm{B}$, et al. Association between villous atrophy in rheumatoid arthritis and a rheumatoid factor and antigliadin specific IgG. Lancet 1988; ii: 819-22.

26 Stenhammar L, Brandt A, Wagermark J. A family study of coeliac disease. Acta Pediatr Scand 1982; 71: 625-8.

27 Corazza GR, Valentini RA, Frisoni M, Volta U, Corrao G, Bianchi FB, et al. Gliadin immune reactivity is associated with overt and latent enteropathy in relatives of coeliac patients. Gastroenterology 1992; 103: 1517-22.

28 Hed J, Lieden E, Ottosson E, Strom M, Wolan A, Groth O, et al. IgA antigliadin antibodies and jejunal mucosal
lesions in healthy blood donors. Lancet 1986; ii: 215 .

29 Mazzetti di Pietralata M, Giorgetti PM, Gregori M, De Simone M, Leonardi C, Barletta PAM, et al. Subclinical coeliac disease. It f Gastroenterol 1992; 24: 352-4.

30 Hill PG, Thomson SP, Holmes GKT. IgA antigliadin antibodies in adult coeliac disease. Clin Chem 1991; 36: 647-50.

31 Volta U, Molinaro N, Fusconi M, Cassani F, Bianchi FB. IgA antiendomysial antibody test. A step forward in celiac disease. Dig Dis Sci 1991; 36: 752-6.

32 Troncone R, Ferguson A. Anti-gliadin antobodies. $f$ Pediatr Gastroenterol Nutr 1991; 12: 150-8. 